\title{
Fall Coloring on Product of Cycles and Powers
}

\author{
S. Chandra Kumar \\ Department of Mathematics, Scott Christian College \\ Nagercoil, Tamil Nadu, India \\ E-mail: kumar.chandra82@yahoo.in \\ T. Nicholas \\ Department of Mathematics, St. Jude's College \\ Thoothoor, Tamil Nadu, India \\ E-mail: nicholas_thadeus@hotmail.com
}

S. Somasundaram

Department of Mathematics, Manonmaniam Sundaranar University

Tirunelveli, Tamil Nadu, India

E-mail: somumsu@rediffmail.com

Received: August 17, 2011 Accepted: September 7, 2011 Published: November 1, 2011

doi:10.5539/jmr.v3n4p152 URL: http://dx.doi.org/10.5539/jmr.v3n4p152

\begin{abstract}
In this paper, we obtain necessary and sufficient conditions for the existence of fall coloring with fall achromatic number $\Delta(G)+1$ in the power of a cycle $C_{n}^{k}$ and in the Cartesian product of two cycles.
\end{abstract}

Keywords: $b$-coloring, Fall achromatic number, Cartesian product of two cycles, Power of a cycle

\section{Introduction}

A $k$-vertex coloring of a graph $G$ is an assignment of $k$ colors $1,2, \ldots, k$, to the vertices. The coloring is proper if no two distinct adjacent vertices share the same color. A graph $G$ is $k$-colorable if $G$ has a proper $k$-vertex coloring. The chromatic number $\chi(G)$ is the minimum number $r$ such that $G$ is $r$-colorable. Each set of vertices colored with one color is an independent set of vertices of $G$, so a coloring is a partition of the vertex set into independent sets. Color of a vertex $v$ is denoted by $c(v)$.

Many graph invariants related to colorings have been defined. Most of them try to minimize the number of colors used to color the vertices under some constraints. For some other invariants, it is meaningful to try to maximize this number. The $b$-chromatic number is such an example. A $b$-coloring is a coloring of the vertices of a graph such that each color class contains a vertex that has a neighbor in all other color classes.(Mostafa Blidia, 2009, p. 1787-1793). Any such vertex is called as a colorful vertex. (Saeed Shaebani, 2009). The $b$-chromatic number $b(G)$ is the largest integer $k$ such that $G$ admits a $b$-coloring with $k$ colors. A fall coloring of a graph $G$ is a proper coloring such that every vertex of $G$ has neighbors in all the other color classes. (Saeed Shaebani, 2009). A fall achromatic coloring is a particular case of $b$-coloring in which every vertex is colorful. (Dunbar, J.E., 2000, p.257-273). We call fall achromatic number, the maximum cardinality of a fall coloring of $G$ which we denote by $\psi_{f}(G)$. Not all graphs are fall colorable. Dunbar et al. have proved that the problem of deciding if a given graph admits a fall coloring is NP-complete. (Dunbar, J.E., 2000, p.257-273)

For a graph $G$, and for any vertex $v$ of $G$, the neighborhood of $v$ is the set $N(v)=\{u \in V(G) /(u, v) \in E(G)\}$ and the degree of $v$ is $\operatorname{deg}(v)=|N(v)| . \Delta(G)$ denotes the maximum degree of a vertex in $G$. Then every graph $G$ satisfies $b(G) \leq \Delta(G)+1$.

A graph is a power of cycle, denoted $C_{n}^{k}$, if $V\left(C_{n}^{k}\right)=\left\{v_{0}\left(=v_{n}\right), v_{1}, v_{2}, \ldots, v_{n-1}\right\}$ and $E\left(C_{n}^{k}\right)=E^{1} \cup E^{2} \cup \ldots \cup E^{k}$, where $E^{i}=\left\{\left(v_{j}, v_{(j+i)(\bmod n)}\right): 0 \leq j \leq n-1\right\}$ and $k \leq\left\lfloor\frac{n-1}{2}\right\rfloor$. (Campos, CN., 2007, p. 585-597) Note that $C_{n}^{k}$ is a $2 k$-regular graph and that $k \geq 1$. We take $\left(v_{0}, \ldots, v_{n-1}\right)$ to be a cyclic order on the vertex set of $G$, and always perform modular operations on edge and vertex indexes. (Campos, CN., 2007, p. 585-597).

A graph $\tilde{G}$ is called covering of $G$ with projection $f: \tilde{G} \rightarrow G$ if there is a surjection $f: V(\tilde{G}) \rightarrow V(G)$ such that 
$\left.f\right|_{N(\tilde{v})}: N(\tilde{v}) \rightarrow N(v)$ is a bijection for any vertex $v \in V(G)$ and $\tilde{v} \in f^{-1}(v)$. (Tamizh Chelvam T., 2009, p. 56-62). In 2001, Lee has introduced a method of studying the domination parameters such as perfect and independent domination through covering projections. (Lee, J., 2001, p. 231-239). He proved the following theorem.

Theorem 1 (Lee, J., 2001, p. 231-239) Let $p: \tilde{G} \rightarrow G$ be a covering projection and let $S$ be a perfect dominating set of $G$. Then $p^{-1}(S)$ is a perfect dominating set of $\tilde{G}$. Moreover, if $S$ is independent, then $p^{-1}(S)$ is independent.

Tamizh Chelvam and Sivagnanam Mutharasu have studied the efficient open dominating sets through covering projections. (Tamizh Chelvam T., 2009, p. 56-62). They proved that the inverse image of an efficient open dominating set under a covering projection is an efficient open dominating set. They proved the following lemma. (Tamizh Chelvam T., 2009 , p. 56-62).

Lemma 2 (Tamizh Chelvam T., 2009, p. 56-62) Let $f: \tilde{F} \rightarrow F$ and $g: \tilde{G} \rightarrow G$ be two covering projections. Then there exists a covering projection $h: \tilde{H} \rightarrow H$, where $\tilde{H}=\tilde{F} \square \tilde{G}$ and $H=F \square G$.

In this paper, we introduced a method of studying fall coloring in graphs through covering projections. We obtain a necessary and sufficient condition for the existence of fall coloring with $\Delta(G)+1$ colors in the power of a cycle $C_{n}^{k}$. Also, we show that the graph $C_{n}^{k}$ is $b$-colorable. Further, we obtain a necessary and sufficient condition for the existence of fall coloring with $\Delta(G)+1$ colors in the Cartesian product of two cycles.

\section{Fall Coloring in $C_{n}^{k}$}

In this section, we obtain a necessary and sufficient condition for the existence of fall coloring with fall coloring number $\Delta(G)+1$ in $C_{n}^{k}$. Further, we illustrate a method of $b$-coloring the graph $C_{n}^{k}$ with $\Delta(G)+1$ colors.

Lemma 3 Let $f: G \rightarrow H$ be a covering projection from a graph $G$ on to another graph $H$. If $H$ has fall coloring number $n$, then so is $G$.

Proof. Assume that $H$ admits a fall coloring with fall achromatic number $n$ and $\left\{H_{1}, H_{2}, \ldots, H_{n}\right\}$ is a color partition of $V(H)$ under $f$. Define $G_{i}=f^{-1}\left(H_{i}\right)$ for $1 \leq i \leq n$. We prove that the graph $G$ admits fall coloring with color classes $G_{1}, G_{2}, \ldots, G_{n}$ and $\psi_{f}(G)=n$.

Since each $H_{i}$ is an independent vertex subset of $H$, by Theorem, each $G_{i}$ is an independent vertex subset of $G$ for $1 \leq i \leq n$. Thus the class $\left\{G_{1}, G_{2}, \ldots, G_{n}\right\}$ is a vertex partition of independent subsets of $G$. It remains to show that each vertex of $G$ is colorful.

Let $u \in V(G)$. Then $v \in G_{i}$ for some $1 \leq i \leq n$. Without loss of generality, assume $i=1$. Then $u \in G_{1}$ and $f(u)=v$ for some $v \in H_{1}$ (by the construction of $G_{1}$ ). Since $v \in H_{1}$ and by the definition of $\left\{H_{1}, H_{2}, \ldots, H_{n}\right\}$, there exist vertices $v_{2}, v_{3}, \ldots, v_{n}$ such that $v_{i} \in H_{i}$ and $\left(v, v_{i}\right) \in E(H)$ for $2 \leq i \leq n$.

Let $f^{-1}\left(v_{i}\right)=u_{i}$ for $2 \leq i \leq n$. Then $u_{i} \in G_{i}$ for $2 \leq i \leq n$. Since $\left.f\right|_{N(u)}: N(u) \rightarrow N(v)$ is a bijection, $N(v) \supseteq\left\{v_{2}, v_{3}, \ldots, v_{n}\right\}$ and $f^{-1}\left(v_{i}\right)=u_{i}$ for $2 \leq i \leq n$, we can conclude that $N(u) \supseteq\left\{u_{2}, u_{3}, \ldots, u_{n}\right\}$. Thus $u$ is adjacent to $u_{i} \in G_{i}$ for $2 \leq i \leq n$. Hence $u$ is a colorful vertex of $G$.

Lemma 4 If $2 k+1$ divides $n$, then the graph $G=C_{n}^{k}$ admits fall coloring with fall achromatic number $\Delta(G)+1$.

Proof. Let $V(G)=\left\{v_{0}, v_{1}, \ldots, v_{n-1}\right\}$ and $E(G)=E^{1} \cup E^{2} \cup \ldots \cup E^{k}$, where $E^{g}=\left\{\left(v_{j}, v_{(j+g)(\bmod n)}\right): 0 \leq j \leq n-1\right\}$. Note that, for each $g$ with $1 \leq g \leq k$, the vertex $v_{j}$ has exactly two neighbors $v_{j \oplus_{n} g}, v_{j \oplus_{n}(n-g)} \in E^{g}$, where $\oplus_{n}$ is the operation, addition modulo $n$. Hence $N\left(v_{j}\right)=\left\{v_{j \oplus_{n} 1}, v_{j \oplus_{n} 2}, \ldots, v_{j \oplus_{n} k}, v_{j \oplus_{n}(n-1)}, v_{j \oplus_{n}(n-2)}, \ldots, v_{j \oplus_{n}(n-k)}\right\}$. Note that $\Delta(G)=2 k$. Let us color the vertices as follows:

For each $j$ with $0 \leq j \leq n-1$, color of the vertex $v_{j}$ is denoted and defined by $c\left(v_{j}\right)=j(\bmod (2 k+1))$. Let $v \in V(G)$. Then $v=v_{i}$ for some integer $i$ with $0 \leq i \leq n-1$ and $c\left(v_{i}\right)=i(\bmod (2 k+1))=g$ for some $0 \leq g \leq 2 k$. Hence $c\left(v_{i \oplus_{n} 1}\right)=g+1(\bmod (2 k+1)), c\left(v_{i \oplus_{n}}\right)=g+2(\bmod (2 k+1)), \ldots, c\left(v_{i \oplus_{n} k}\right)=g+k(\bmod (2 k+1)), c\left(v_{i \oplus_{n}(n-k)}\right)=g+k+$ $1(\bmod (2 k+1)), c\left(v_{i \oplus_{n}(n-(k-1))}\right)=g+k+2(\bmod (2 k+1)), \ldots, c\left(v_{i \oplus_{n}(n-1)}\right)=g+2 k(\bmod (2 k+1))$.

Thus the vertex $v_{i}$ is a colorful vertex of $G$ and the above coloring is a proper coloring of $G$. Hence $G$ is fall colorable with $2 k+1$ colors.

Lemma 5 If the graph $G=C_{n}^{k}$ is fall colorable with $\Delta(G)+1$ colors, then $2 k+1$ divides $n$.

Proof. Assume that $G$ is fall colorable with $\Delta(G)+1=2 k+1$ colors, namely $0,1,2, \ldots, 2 k$. Suppose $2 k+1$ does not divide $n$. Then $n=i(2 k+1)+j$ for some positive integers $i, j$ with $1 \leq j \leq 2 k$. 
Consider the vertex $v_{0}$. Without loss of generality, assume that $c\left(v_{0}\right)=0$. Since $G$ is fall colorable with $2 k+1$ colors and $N\left(v_{0}\right)=\left\{v_{1}, v_{2}, \ldots, v_{k}, v_{n-1}, v_{n-2}, \ldots, v_{n-k}\right\}$, we should color all these vertices with different colors among the colors $1,2, \ldots, 2 k$. Without loss of generality, assume the following: $c\left(v_{1}\right)=1, c\left(v_{2}\right)=2, \ldots, c\left(v_{k}\right)=k, c\left(v_{n-k}\right)=k+1$, $c\left(v_{n-(k-1)}\right)=k+2, \ldots, c\left(v_{n-1}\right)=2 k$.

Consider the vertex $v_{1}$. Note that $N\left(v_{1}\right)=\left\{v_{2}, v_{3}, \ldots, v_{k+1}, v_{0}, v_{n-1}, v_{n-2}, \ldots, v_{n-(k-1)}\right\}$ and all the neighbors of $v_{1}$ except the vertex $v_{k+1}$ are colored with different colors, namely $0,2,3, \ldots, k, k+2, k+3, \ldots, 2 k$. Also $c\left(v_{1}\right)=1$. Hence the vertex $v_{k+1}$ must be colored with the color $k+1$. That is $c\left(v_{k+1}\right)=k+1$.

Similarly, one can obtain the following: $c\left(v_{k+2}\right)=k+2, c\left(v_{k+3}\right)=k+3, \ldots, c\left(v_{2 k}\right)=2 k, c\left(v_{2 k+1}\right)=0, c\left(v_{(2 k+1)+1}\right)=1$, $c\left(v_{(2 k+1)+2}\right)=2, \ldots, c\left(v_{(2 k+1)+2 k}\right)=2 k, c\left(v_{2(2 k+1)}\right)=0, c\left(v_{2(2 k+1)+1}\right)=1$ and so on.

Hence, we must have $c\left(v_{i(2 k+1)}\right)=0, c\left(v_{i(2 k+1)+1}\right)=1, c\left(v_{i(2 k+1)+2}\right)=2, \ldots, c\left(v_{i(2 k+1)+j}\right)=c\left(v_{n}\right)=c\left(v_{0}\right)=j$, where $j \neq 0$, a contradiction to the fact that $c\left(v_{0}\right)=0$.

From Lemma and Lemma, one can derive the following theorem which gives a necessary and sufficient condition for the existence of fall coloring with $\Delta(G)+1$ colors in the graph $C_{n}^{k}$.

Theorem 6 The graph $C_{n}^{k}$ is fall colorable with $\Delta(G)+1$ colors if and only if $2 k+1$ divides $n$.

It is conjectured that every $d$-regular graph with girth at least 5 has a $b$-coloring with $d+1$ colors. (El-Sahili, A., 2006). Mostafa Blidia, Frederic Maffray and Zoham Zemira showed that the Petersen graph infirms this conjecture, and they propose a new formulation of this question and give a positive answer for small degree as given below. (Mostafa Blidia, 2009, p. 1787-1793).

Theorem 7(Mostafa Blidia, 2009, p. 1787-1793) Let $G$ be a d-regular graph with girth $g(G) \geq 5$, different from the Petersen graph, and with $d \leq 6$. Then $b(G)=d+1$.

Further they proposed the following conjecture.

Conjecture: Every $d$-regular graph with girth at least 5, different from the Petersen graph, has a $b$-coloring with $d+1$ colors.

In the next lemma, we prove that a $2 k$-regular graph $C_{n}^{k}$ is $b$-colorable with $2 k+1$ colors. Note that the girth of $C_{n}^{k}$ is 3 when $k \geq 2$.

Lemma 8 The graph $C_{n}^{k}$ is b-colorable with $2 k+1$ colors.

Proof. Let $V(G)=V\left(C_{n}^{k}\right)=\left\{v_{0}, v_{1}, \ldots, v_{n-1}\right\}$ and $E\left(C_{n}^{k}\right)=E^{1} \cup E^{2} \cup \ldots \cup E^{k}$, where $E^{g}=\left\{\left(v_{j}, v_{(j+g)(\bmod n)}\right): 0 \leq j \leq n-1\right\}$. By division algorithm, one can write $n=h(2 k+1)+j$ for some positive integers $j, h$ with $0 \leq j \leq 2 k$ and $h=\left\lfloor\frac{n}{2 k+1}\right\rfloor$.

Case 1: Suppose $1 \leq j \leq k$. Color the vertices as follows:

For $0 \leq g \leq h(2 k+1)$, color of the vertex $v_{g}$ as $c\left(v_{g}\right)=g(\bmod (2 k+1))$ and $c\left(v_{h(2 k+1)+1}\right)=k+1, c\left(v_{h(2 k+1)+2}\right)=$ $k+2, \ldots, c\left(v_{h(2 k+1)+(j-1)}\right)=k+j-1$.

Case 2: Suppose $k+1 \leq j \leq 2 k+1$. Color the vertices as follows:

For $0 \leq g \leq h(2 k+1)$, color of the vertex $v_{g}$ as $c\left(v_{g}\right)=g(\bmod (2 k+1))$ and $c\left(v_{h(2 k+1)+1}\right)=1, c\left(v_{h(2 k+1)+2}\right)=$ $2, \ldots, c\left(v_{h(2 k+1)+(j-1)}\right)=j-1$.

One can easily verify that in both the cases, the above colorings are $b$-colorings of $C_{n}^{k}$ with $2 k+1$ colors.

\section{Fall coloring on Cartesian product of two cycles with achromatic number $\Delta+1$}

The Cartesian product $G \square H$ of two graphs $G$ and $H$, is the graph with vertex set $V(G \square H)=V(G) \times V(H)$ and edge set $E(G \square H)=\left\{\left(\left(x_{1}, y_{1}\right),\left(x_{2}, y_{2}\right)\right):\left(x_{1}, x_{2}\right) \in E(G)\right.$ with $y_{1}=y_{2}$ or $\left(y_{1}, y_{2}\right) \in E(H)$ with $\left.x_{1}=x_{2}\right\}$. (Haynes, T.W., 2000). In this section, a necessary and sufficient condition for the existence of fall coloring with $\Delta(G)+1$ colors in the Cartesian product of two cycles has been obtained. The vertex set of the cycle $C_{i}$ is taken as $V\left(C_{i}\right)=\{0,1, \ldots,(i-1)\}$.

Remark 9 When $V\left(C_{5}\right)=\{0,1,2,3,4\}$, the sets $A_{1}, A_{2}, A_{3}, A_{4}$ and $A_{5}$ forms a vertex partition of independent subsets of $V\left(C_{5} \square C_{5}\right)$, where $A_{1}=\{(1,1),(2,3),(3,0),(4,2),(0,4)\}, A_{2}=\{(2,1),(3,3),(4,0),(0,2),(1,4)\}, A_{3}=\{(0,1),(1,3),(2,0)$, $(3,2),(4,4)\}, A_{4}=\{(1,2),(2,4),(3,1),(4,3),(0,0)\}$ and $A_{5}=\{(1,0),(2,2),(3,4),(4,1),(0,3)\}$.

Lemma 10 Let $m, n$ be integers which are multiples of 5. Then the graph $G=C_{m} \square C_{n}$ is fall colorable with fall achromatic number $\Delta(G)+1$. 
Proof. Let $m, n$ be integers which are multiples of 5 . Note that $\Delta(G)+1=5$.

Claim 1: The graph $C_{5} \square C_{5}$ is fall colorable with 5 colors.

Consider the vertex subsets $A_{1}, A_{2}, A_{3}, A_{4}$ and $A_{5}$ as given in Remark .

Color the vertices of $C_{5} \square C_{5}$ as follows: For each $i$ with $1 \leq i \leq 5, c(v)=i$ if and only if $v \in A_{i}$. Since $A_{i}$ 's are independent, to prove the claim, it is enough to prove that each vertex is colorful.

Note that, for $(a, b) \in V\left(C_{5} \square C_{5}\right), N((a, b))=\left\{\left(a \oplus_{5} 1, b\right),\left(a \oplus_{5} 4, b\right),\left(a, b \oplus_{5} 1\right),\left(a, b \oplus_{5} 4\right)\right\}$. Consider the vertex $(1,1) \in A_{1}$. It is adjacent with the vertices $(2,1) \in A_{2},(0,1) \in A_{3},(1,2) \in A_{4}$ and $(1,0) \in A_{5}$. Hence $(1,1)$ is a colorful vertex of $C_{5} \square C_{5}$. Similarly, one can easily verify that each other vertex of $C_{5} \square C_{5}$ is a colorful vertex of $C_{5} \square C_{5}$ under the above coloring.

Claim 2: The graph $C_{m} \square C_{n}$ is fall colorable with fall achromatic number 5 .

Define $f: V\left(C_{m}\right) \rightarrow V\left(C_{5}\right)$, by $f(x)=x(\bmod 5)$ for all $x \in V\left(C_{m}\right)$ and $g: V\left(C_{n}\right) \rightarrow V\left(C_{5}\right)$, by $g(x)=x(\bmod 5)$ for all $x \in V\left(C_{n}\right)$. Then $f$ and $g$ are covering projections respectively from $C_{m}$ and $C_{n}$ onto the graph $C_{5}$. Then by Lemma, there exists a covering projection from $C_{m} \square C_{n}$ onto the graph $C_{5} \square C_{5}$. By Claim 1 and by Lemma, one can conclude that the graph $G=C_{m} \square C_{n}$ is fall colorable with $\Delta(G)+1$ colors.

Lemma 11 Let $m, n \geq 5$ be integers. Suppose the graph $C_{m} \square C_{n}$ is fall colorable with fall achromatic number $\Delta(G)+1$, then $m$ and $n$ are multiples of 5 .

Proof. Suppose the graph $C_{m} \square C_{n}$ is fall colorable with 5 colors. Since each vertex of $C_{m} \square C_{n}$ is adjacent with exactly four vertices, all these adjacent vertices must be colored with different colors.

In this lemma, by five consecutive vertices of $C_{m} \square C_{n}$, we mean that $\left\{\left(a, b \oplus_{n} i\right): 0 \leq i \leq 4\right\}$.

Claim 1: Any five consecutive vertices receive different colors.

On the contrary, assume that there are two vertices $x$ and $y$ in a set of five consecutive vertices have the same color.

[Figure 1]

Case 1: Suppose $x=\left(a, b \oplus_{n} i\right)$ and $y=\left(a, b \oplus_{n}(i+1)\right)$ for $0 \leq i \leq 3$. Then the coloring is not a proper coloring.

Case 2: Suppose $x=\left(a, b \oplus_{n} i\right)$ and $y=\left(a, b \oplus_{n}(i+2)\right)$ for $0 \leq i \leq 2$. In this case, the two neighbors of the vertex $\left(a, b \oplus_{n}(i+1)\right)$, namely $x y$ will have the same color and so the vertex $\left(a, b \oplus_{n}(i+1)\right)$ is not a colorful vertex.

Case 3: Suppose $x=\left(a, b \oplus_{n} i\right)$ and $y=\left(a, b \oplus_{n}(i+3)\right)$ for $0 \leq i \leq 1$.

Without loss of generality, assume $x=(a, b)$ and $y=\left(a, b \oplus_{n} 3\right)$ as shown in Figure 1. Without loss of generality, assume that $c(x)=c(y)=1$.

Consider the vertex $d$ and their uncolored neighboring vertices $b, e$ and $g$. Without loss of generality, assume that $c(d)=2$, $c(b)=4, c(e)=3$ and $c(g)=5$.

Consider the vertex $c$. Obviously, we can not use colors 3 and 4 to color the vertex $c$. If $c(c)=2$, then the vertex $b$ is not a colorful vertex, a contradiction. If $c(c)=1$, then $i$ is not a colorful vertex, a contradiction. Hence $c(c)=5$.

Now, consider the vertex $h$. Clearly, we can not use the colors 3 and 5 to color the vertex $h$. If $c(h)=2$, then the vertex $g$ is not a colorful vertex. If $c(h)=1$, then the vertex $e$ is not a colorful vertex. Hence $c(h)=4$.

Consider the vertex $f$. Clearly, we can not use the colors 1 and 5 to color the vertex $f$. If $c(f)=2$ or 4 , then the vertex $g$ is not a colorful vertex. Hence $c(f)=3$.

Now, consider the vertex $a$. We cannot use the colors $1,2,3,4$ and 5 to color the vertex $a$, a contradiction to the fact that $C_{m} \square C_{n}$ is fall colorable with 5 colors.

Case 4: Suppose $x=(a, b)$ and $y=\left(a, b \oplus_{n} 4\right)$ as shown in Figure 2. Assume that $c(x)=c(y)=1$.

By Case 1 and Case 2, we cannot use the color 1 to color the vertices $d$, $e$ and $f$. Consider the vertex $e$ and their uncolored neighboring vertices $b, d, f$ and $h$. Without loss of generality, assume that $c(b)=5, c(d)=4, c(f)=3$ and $c(h)=1$.

Consider the vertex $c$. Obviously, we can not use the colors 3 and 5 to color the vertex $c$. If $c(c)=1$, then the vertex $j$ is not a colorful vertex, a contradiction. If $c(c)=2$, then $b$ is not a colorful vertex, a contradiction. Hence $c(c)=4$. 
Now consider the vertex $i$. Clearly we can not color the vertex $i$ with colors 1 and 3 . Also, when $c(i)=2$, the vertex $h$ will not be a colorful vertex and when $c(i)=4$, the vertex $f$ will not be a colorful vertex. Hence $c(i)=5$.

Consider the vertex $g$. Clearly we can not color the vertex $g$ with colors 1 and 4 . Also, when $c(g)=2$ or 5 , the vertex $h$ will not be a colorful vertex. Hence $c(g)=3$.

Now, consider the vertex $a$. We cannot use the colors 1,2,3,4 and 5 to color the vertex $a$, a contradiction to the fact that $C_{m} \times C_{n}$ is fall colorable with 5 colors.

Thus in all the cases, we get a contradiction and hence Claim 1 is true. Hence $n$ must be a multiple of 5 .

[Figure 2]

Similarly, by considering $\left\{\left(a \oplus_{m} i, b\right): 0 \leq i \leq 4\right\}$ as five consecutive vertices, one can prove that any five consecutive vertices receive different colors and hence $m$ is also a multiple of 5 .

From Lemma and Lemma, one can conclude the following theorem.

Theorem 12 Let $m, n \geq 5$ be integers. Then the graph $C_{m} \square C_{n}$ is fall colorable with $\Delta(G)+1$ colors if and only if 5 divides $m$ and $n$.

\section{Acknowledgment}

The authors would like to thank Dr. M.S.Mutharasu, Assistant Professor, Department of Mathematics, Sri Krishna College of Engineering and Technology, Coimbatore, India, for having helped us in the proof reading of this article.

\section{References}

Campos, C. N. \& De Mello C. P. (2007). A result on the total coloring of powers of cycles. Discrete Applied Mathematics, 155, 585-597. http://dx.doi.org/10.1016/j.dam.2006.08.010

Dunbar, J. E., Hedetniemi, S. M., Hedetniemi Stephen T., Jacobs, D.P., Knisely, D.J., Renu Laskar \& Douglas F. Rall. (2000). Fall colorings of graphs. J. of Combin. Math. and Combin. Computing, 33, 257-273.

El-Sahili, A. \& Kouider, M. (2006). About b-colorings of regular graphs. Res. Rep. 1432, LRI, Univ. Orsay, France.

Gary Chartrand \& Ping Zhang. (2009). Chromatic Graph Theory. New York:CRC Press.

Haynes, T. W., Hedetniemi, S. T. \& Slater, P. J. (2000). Fundamentals of Domination in Graphs. Marcel Dekker.

Lee, J. (2001). Independent perfect domination sets in Cayley graphs. J. Graph Theory, 37(4), 231-239. http://dx.doi.org/ $10.1002 /$ jgt. 1016

Mostafa Blidia, Frederic Maffray \& Zoham Zemira. (2009). On b-colorings in regular graphs, Discrete Applied Mathematics, 157, 1787-1793. http://dx.doi.org/10.1016/j.dam.2009.01.007

Saeed Shaebani. (2009). On Fall Colorings of Kneser Graphs. [Online] Available: http://arxiv.org/abs/0909.2772.

Tamizh Chelvam, T. \& Sivagnanam Mutharasu. (2009). Efficient Domination in Bi-Cayley Graphs. International Journal of Mathematical Combinatorics, 4, 56-62. 


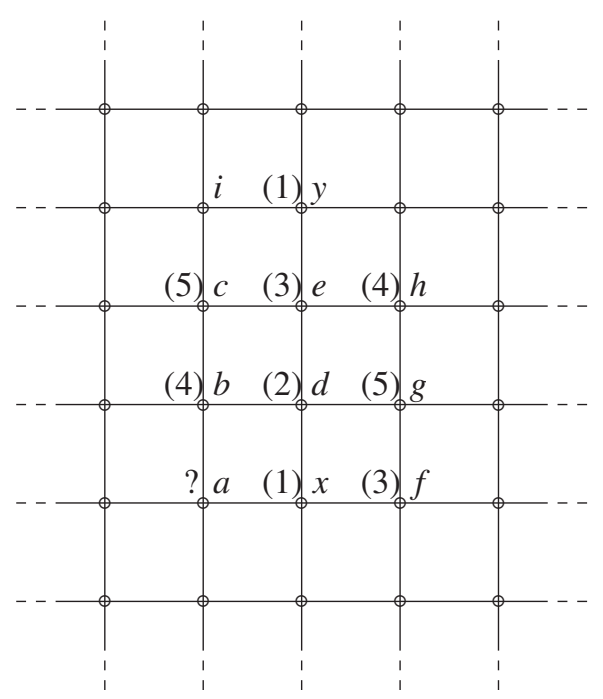

Figure 1

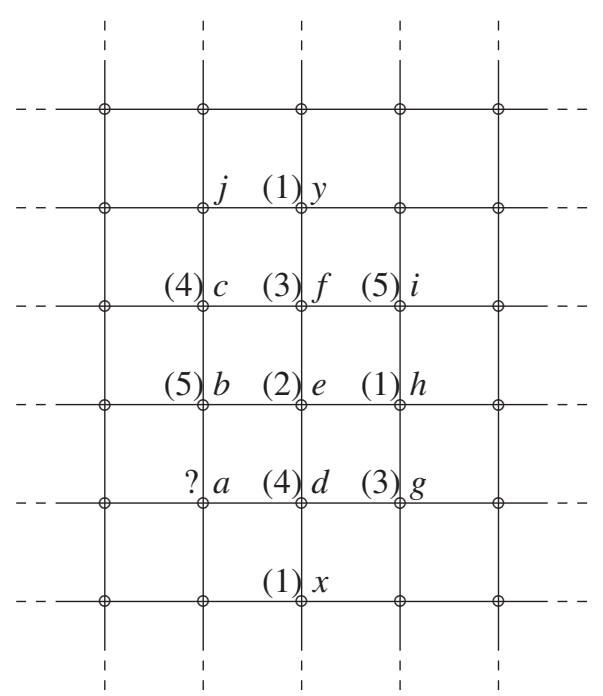

Figure 2 